\title{
Protracted low doses of temozolomide for the treatment of patients with recurrent glioblastoma: A phase II study
}

\author{
MATTEO SANTONI ${ }^{1}$, ALESSANDRO PACCAPELO ${ }^{1}$, LUCIANO BURATTINI ${ }^{1}$, MARISTELLA BIANCONI ${ }^{1}$, \\ MASSIMO CARDINALI ${ }^{2}$, LETIZIA FABBIETTI ${ }^{2}$, ROBERTO TRIGNANI $^{3}$, \\ FRANCO RYCHLICKI ${ }^{3}$ and STEFANO CASCINU ${ }^{1}$ \\ ${ }^{1}$ Department of Medical Oncology, ${ }^{2}$ Radiotherapy Unit and ${ }^{3}$ Division of Neurosurgery, \\ Polytechnic University of the Marche Region, 60126 Torrette, Ancona, Italy
}

Received March 2, 2012; Accepted June 13, 2012

DOI: $10.3892 / \mathrm{ol} .2012 .788$

\begin{abstract}
O}^{6}$-alkylguanine-DNA alkyltransferase (AGAT), involved in temozolomide-induced DNA damage repair, plays a key role in the efficacy of temozolomide. AGAT activity may be reduced by protracted temozolomide doses. On the basis of the preclinical findings, we treated patients with a histologically-proven diagnosis of glioblastoma (GBM) following adjuvant temozolomide failure with a low protracted dose of temozolomide $\left(130 \mathrm{mg} / \mathrm{m}^{2} /\right.$ day, days $1-7$ and $15-21$, every 4 weeks). The primary endpoint of the study was 6-month progression-free survival (PFS-6 m). The secondary endpoints were overall survival (OS) from the start of temozolomide alternative schedule and toxicity. Enrolment was ceased at 27 patients due to the lack of effectiveness of this regimen. Results indicate that our schedule is well-tolerated, but ineffective in patients with GBM and further strategies are required to improve the outcome of these patients.
\end{abstract}

\section{Introduction}

Although temozolomide is considered to be the backbone in the treatment of brain tumors, the results are not particularly favorable. Its efficacy is limited by the presence of intrinsic or acquired resistance mechanisms (1). Temozolomide exerts its activity by DNA methylation at the $\mathrm{N}^{7}$ and $\mathrm{O}^{6}$ positions of guanine and at the $\mathrm{O}^{3}$ position of adenine (2). The O6-methylguanine-DNA methyltransferase (MGMT) gene is located on chromosome 10q26 and encodes a DNA repair enzyme, $\mathrm{O}^{6}$-alkylguanine-DNA-alkyltransferase (AGAT), that counteracts the effects of alkylating cytotoxic drugs by removing methyl adducts from the $\mathrm{O}^{6}$ position of guanine.

Correspondence to: Dr Matteo Santoni, Department of Medical Oncology, Polytechnic University of the Marche Region, 71 Via Conca, 60126 Torrette, Ancona, Italy

E-mail: mattymo@alice.it

Key words: clinical trials, glioblastoma, low dose temozolomide, progression-free survival, overall survival
In malignant gliomas, the $M G M T$ gene is often inactivated due to aberrant methylation of its promoter region (3). Thus, MGMT promoter methylation status has become clinically relevant as a molecular marker associated with response to alkylating chemotherapy and survival of glioblastoma (GBM) patients (4). Protracted exposure to an alkylating agent may inactivate enzyme activity by saturating the AGAT available copies and newly synthesized molecules, overcoming the inherent resistance of glioma cells (5).

A phase I study demonstrated that a protracted low-dose temozolomide schedule $\left(75 \mathrm{mg} / \mathrm{m}^{2} /\right.$ day, for 21 days, every 28 days) was well-tolerated with a dose-limiting toxicity by thrombocytopenia. The maximum tolerated dose was $100 \mathrm{mg} / \mathrm{m}^{2}(6)$.

Based on the data, we assessed the effects of a prolonged temozolomide schedule $\left(130 \mathrm{mg} / \mathrm{m}^{2} /\right.$ day on days $1-7$ and $\left.15-21\right)$ in GBM patients following adjuvant temozolomide failure.

\section{Materials and methods}

Patients. The study was originally designed for 50 patients, however, only 27 patients were enrolled. We treated consecutive recurrent GBM patients following chemoradiation treatment with temozolomide and a sequentially failing adjuvant temozolomide schedule $\left(200 \mathrm{mg} / \mathrm{m}^{2} /\right.$ day, days $1-5$, every 4 weeks) (7). All patients had undergone previous surgery followed by standard radiotherapy (60 Gy/30 fractions). Patient characteristics are summarized in Table I. This study was approved by the ethics committee of the Polytechnic University of the Marche Region. Informed patient consent was obtained either from the patient or the patient's family

Chemotherapy. Chemotherapy cycles consisted of temozolomide $130 \mathrm{mg} / \mathrm{m}^{2} /$ day, days $1-7$ and $15-21$, every 4 weeks, for a maximum of 1 year. No dose escalation was allowed and patients were fasted for at least $2 \mathrm{~h}$ prior to and following temozolomide administration. Antiemetic prophylaxis was provided by metoclopramide or a 5-hydroxytriptamine antagonist.

Patients were closely monitored for toxicity throughout the cycles and all adverse events were recorded and graded according to the common toxicity criteria of the National 
Table I. Characteristics of 27 recurrent GBM patients.

\begin{tabular}{lc}
\hline Patient characteristics & No. \\
\hline Gender & \\
Female & 10 \\
Male & 17 \\
Age (years) & \\
Median & 57.82 \\
Range & $32.40-73.44$ \\
Karnofsky performance status & 80 \\
Median & $70-100$ \\
Range & \\
Surgery & 27 \\
Undergone & \\
Extent of resection & 23 \\
Gross total resection & 4 \\
Partial resection or biopsy & \\
\hline
\end{tabular}

GBM, glioblastoma.

Cancer Institute version 3.0. Hematologic controls were conducted weekly, while complete biochemistry was assessed once per cycle, preferably on day 14 .

Progression-free survival (PFS) and 6-month PFS (PFS-6 m) were evaluated from the start of low-dose temozolomide to progression, and the median overall survival (OS) was calculated from the start of low-dose temozolomide until mortality from any cause. In this intent-to-treat study, PFS, PFS-6 m and OS were calculated using the Kaplan-Meier method (8).

The response was determined by CT, MRI and clinical examination. The Macdonald criteria (9) were selected to evaluate the MRI. Partial response (PR) was defined as a greater than $50 \%$ decrease in the area of contrast enhancement and a complete response (CR) was defined as the disappearance of all target lesions. Disease progression (DP) was defined as an increase of greater than $25 \%$ in the area of contrast enhancement, the appearance of new lesions and the deterioration of a patient's clinical status. Finally, stable disease (SD) included patients with no DP, as well as no RP or CR criteria. Patients were withdrawn if they had progressive disease, unacceptable toxicity or retracted their consent.

Statistical analysis. The study was designed according to the Simon two-stage design $(\mathrm{P} 0=0.10, \mathrm{P} 1=0.25, \mathrm{a}=0.10$ and $\mathrm{b}=0.10)$. Statistical analysis was performed with MedCalc, version 11.4.4.0 (MedCalc Software, Mariakerke, Belgium). $\mathrm{P}<0.05$ was considered to indicate a statistically significant difference.

\section{Results}

Enrolment was ceased at 27 patients due to the ineffectiveness of this schedule for patients with GBM. A total of 193 treatment cycles were performed (median, 6 cycles; range, 2-26 cycles) for each patient. Additionally, the
Table II. Results of a low protracted temozolomide schedule in GBM patients following adjuvant temozolomide failure.

\begin{tabular}{lc}
\hline Results & No. \\
\hline Median number of cycles & 6 \\
Patients with CR (\%) & $0 / 27(0)$ \\
Patients with PR (\%) & $3 / 27(11.1)$ \\
Patients with SD (\%) & $5 / 27(18.5)$ \\
Patients with DP (\%) & $19 / 27(70.4)$ \\
Median OS (months) & 24.7 \\
Median OS from chemotherapy (months) & 6.9 \\
Median PFS (months) & 4.3 \\
6-month PFS (\%) & $33.3 \%$
\end{tabular}

GBM, glioblastoma. CR, complete response; PR, partial response; $\mathrm{SD}$, stable disease; DP, disease progression; OS, overall survival; PFS, progression-free survival.

PFS-6 m rate was $33.33 \%$ (median PFS, 4.34 months; range, 4.32-7.36 months) and the OS from the start of low-dose temozolomide chemotherapy was 6.90 months (range, ?-7.36 months). Among the 20 evaluable GBM patients, 3 (11.11\%) PR, 5 (18.51\%) SD and 0 CR cases were observed. The most common reason for drug discontinuation was DP. The results are summarized in Table II.

The low-dose temozolomide schedule was well-tolerated, with no observed grade 4 adverse events (AE). Increased transaminases and lymphopenia were observed in $15 \%$ (grade $3 \mathrm{AE}, 5 \%$ ) and $25 \%$ (grade $3 \mathrm{AE}, 15 \%$ ) of these patients, respectively.

\section{Discussion}

In previous studies investigating temozolomide regimens the PFS- 6 m ranged from $18-24 \%$. As the antitumor activity of temozolomide depends on the level of AGAT within the tumor cells, the depletion of AGAT via a continuous dosing schedule was proposed in order to improve the activity of temozolomide in several trials (5). In a phase II study, a continuous temozolomide administration $\left(75 \mathrm{mg} / \mathrm{m}^{2} /\right.$ day, over a 6-week period, with a 4-week break) achieved a PFS-6 m of $19 \%$, a median PFS of 2.3 months and no objective responses in 28 pretreated GBM patients. Although this schedule allowed a higher dose intensity $\left(1260 \mathrm{mg} / \mathrm{m}^{2}\right)$ compared to the standard schedule $\left(1000 \mathrm{mg} / \mathrm{m}^{2}\right)$ and demonstrated a good tolerability, it did not result in any improvement in patients with recurrent GBM. Similar results were obtained by Wick et al (10) in 90 GBM patients with a different 28-day continuous temozolomide schedule $\left(150 \mathrm{mg} / \mathrm{m}^{2}\right.$ delivered for 1-week on/1 week off).

In the present study, following adjuvant temozolomide failure patients received temozolomide at a dose of $130 \mathrm{mg} / \mathrm{m}^{2} /$ day on days $1-7$ and $15-21$ every 4 weeks. We observed poor results in terms of the PFS- $6 \mathrm{~m}$ rate, median PFS and median OS from the start of low-dose temozolomide. Toxicity was mild and no grade $4 \mathrm{AE}$ were observed. 
In conclusion, our findings indicate that a prolonged low-dose temozolomide schedule is well-tolerated, but ineffective in patients with GBM. Further strategies are required to improve the outcome of these patients.

\section{Acknowledgements}

The authors offer sincere gratitude to supervisors, Dr Rossana Berardi and Dr Mario Scartozzi, for their support throughout this study. Particular thanks are due to Dr Luciano Burattini for his endorsement.

\section{References}

1. Reardon DA, Rich JN, Friedman HS and Bigner DD: Recent advances in the treatment of malignant astrocytoma. J Clin Oncol 24: 1253-1265, 2006.

2. Denny BJ, Wheelhouse RT, Stevens MF, Tsang LL and Slack JA: NMR and molecular modeling investigation of the mechanism of activation of the antitumor drug temozolomide and its interaction with DNA. Biochemistry 33: 9045-9051, 1994.
3. Zhang J, Stevens MF, Laughton CA, Madhusudan S and Bradshaw TD: Acquired resistance to temozolomide in glioma cell lines: molecular mechanisms and potential translational applications. Oncology 78: 103-114, 2010.

4. Riemenschneider MJ, Hegi ME and Reifenberger G: MGMT promoter methylation in malignant gliomas. Target Oncol 5: 161-165, 2010.

5. Tolcher AW, Gerson SL, Denis L, et al: Marked inactivation of O6-alkylguanine-DNA alkyltransferase activity with protracted temozolomide schedules. Br J Cancer 88: 1004-1011, 2003.

6. Brandes AA, Tosoni A, Cavallo G, et al: Temozolomide 3 weeks on and 1 week off as first-line therapy for recurrent glioblastoma: phase II study from gruppo italiano cooperativo di neuro-oncologia (GICNO). Br J Cancer 95: 1155-1160, 2006.

7. Stupp R, Mason WP, van den Bent MJ, et al: Radiotherapy plus concomitant and adjuvant temozolomide for glioblastoma. N Engl J Med 352: 987-996, 2005.

8. Kaplan E and Meier P: Non parametric estimation from incomplete observations. J Am Stat Assoc 53: 457-481, 1958.

9. Macdonald DR, Cascino TL, Schold SC Jr and Cairncross JG Response criteria for phase II studies of supratentorial malignant glioma. J Clin Oncol 8: 1277-1280, 1990.

10. Wick A, Felsberg J, Steinbach JP, et al: Efficacy and tolerability of temozolomide in an alternating weekly regimen in patients with recurrent glioma. J Clin Oncol 25: 3357-3361, 2007. 